DrLuyten's suggestion concerning catalogues of orbits of visual and spectroscopic binaries was discussed. The following recommendation to the General Assembly was passed: "That the Union recommend that an observatory publish at frequent intervals complete catalogues of all known orbits of visual and spectroscopic binaries."

Dr Parvulesco recommended the use of a certain standard system of projection for studying the galactic distribution of celestial objects.

It was agreed to recommend to the General Assembly the adoption of the galactic pole $\alpha=\mathrm{r} 2^{\mathrm{h}} 4 \mathrm{O}^{\mathrm{m}}, \delta=+28^{\circ}$ ( $\mathrm{rgOO}$ ) as a standard for computing galactic co-ordinates.

\title{
COMMISSION 34. (SOLAR PARALLAX.)
}

\author{
President: Di Spencer Jones. \\ Secretary: Prof. F. Slocum.
}

The President first summarized the present position of the work of determining the solar parallax as given in the Report.

In connection with the determination of the positions of the reference stars it was stated that within the next six months or so the reductions of all the series of photographs will be completed and it will then be possible to co-ordinate the results and publish a definitive list of secondary comparison stars. At the same time it has become apparent that there are appreciable errors in the positions based on the meridian observations of some of the primary stars given in Prof. Kopff's catalogue. By co-ordinating the measures of the several series of path plates it will be possible to derive corrections to the positions of the Kopff stars, which will still keep them on the system adopted by Prof. Kopff, but will improve their homogeneity.

The second portion of the work of the Commission concerns the ephemeris of Eros. This work is being carried on by Prof. Witt with the aid of Dr von Schelling. Supplementing the statements in the Report, Prof. Witt states that he finds a regular run in the differences between calculation and observation, and he thinks it is, therefore, necessary to include a correction to the mass of the earth as an additional unknown in the solution. The demi-definitive ephemeris, which has been published in the Astronomische Abhandlungen, is sufficiently accurate for the purpose of deriving the solar parallax by the decimal method but is scarcely of the accuracy needed for the derivation of the mass of the Moon. It is desirable that the ephemeris should be improved by working backwards and connecting on to previous observations by applying general perturbations. Prof. Witt is working along this line and hopes that funds will be available for the continued employment of Dr von Schelling as computer.

From the large number of observations taken around the opposition of I93I, the solar parallax, by the diurnal method or by observations made almost simultaneously at different stations, will be derived with a very small probable error, but it is possible that systematic errors, due to differences in colour of Eros and the comparison stars, etc., may be appreciably larger than the internal errors. The best determination of the solar parallax may ultimately come from the gravitational method along the lines developed by Prof. Noteboom. Therefore the continuance of the investigations of Prof. Witt to obtain the most accurate determination of the orbit of Eros is a piece of work that should be supported by this Commission. 
After comments and discussion by Messrs Jackson, Jones, Slocum, van Maanen, Gregory apd Verbaandert, two resolutions were adopted.

The first was proposed by Dr Jackson.

"Commission 34 recommends that no programme for the determination of solar parallax should be undertaken at the opposition of Eros in I937, but urges observatories in the northern hemisphere to obtain positions of Eros with instruments comparable in focal length to the astrographic telescopes, and with meridian circles, over as long an arc as possible."

The second resolution was proposed by Prof. Stroobant.

"Commission 34 would welcome a grant of $£$ Ioo for its work during the next three years, to be used to assist Dr Witt in his investigation of the orbit of Eros."

\title{
COMMISSION 35. (STELLAR CONSTITUTION.)
}

\author{
President: Sir ARThur EDdington. \\ SECRETARY: Prof. H. N. RusSELl.
}

The Commission spent an hour in a general discussion of problems of stellar constitution. The report as printed was unanimously adopted.

\section{COMMISSION 36. (SPECTROPHOTOMETRY.)}

President: Prof. H. H. Plaskett.

SECRETARY: Dr R. O. REDMAN.

In view of the wide range of topics covered by the Commission, it was agreed that it would be desirable to press for the nomination of a new president for the Commission each three years.

\section{Report}

In a discussion of stellar "temperatures" (§ 2.I) Prof. Russell referred to the importance of "excitation temperatures", as determined from the ratio of numbers of atoms in two states of different energy in the atom. Dr Minnaert pointed out that this was essentially only a "brightness temperature" to which Prof. Russell agreed, but added that in this way "brightness temperatures" could be found for those stars for which no measures of angular diameter were available. Dr Kienle and Mr Greaves jointly made the following suggestions on "stellar temperature" designations:

I. The designation "effective temperature" should no longer be used for temperatures which are really only "colour temperatures". The symbol $T_{e}$ should be reserved for true effective temperatures, defined as the temperature of a full radiator ("black body") which emits the same amount of total radiation as the star.

2. All that can be done by spectrophotometric observations of the continuous spectrum of the stars is to derive relative monochromatic magnitudes. Observers should therefore be requested to publish their observations either in the form of relative monochromatic magnitudes $\Delta m_{\lambda}=m_{\lambda}{ }^{*}-m_{\lambda}{ }^{0}$,

or relative gradients $G=0.92 \mathrm{I} \frac{d \Delta m_{\lambda}}{d \mathrm{I} / \lambda}$, 